\title{
Criminologie
}

\section{Suicide et tentatives de suicide en prison : vulnérabilité, ostracisme et soutien social}

\section{Joel Harvey et Alison Liebling}

Volume 34, numéro 2, automne 2001

Comportements suicidaires et délinquance

URI : https://id.erudit.org/iderudit/027505ar

DOI : https://doi.org/10.7202/027505ar

Aller au sommaire du numéro

\section{Éditeur(s)}

Les Presses de l'Université de Montréal

ISSN

0316-0041 (imprimé)

1492-1367 (numérique)

Découvrir la revue

Citer cet article

Harvey, J. \& Liebling, A. (2001). Suicide et tentatives de suicide en prison : vulnérabilité, ostracisme et soutien social. Criminologie, 34(2), 57-83.

https://doi.org/10.7202/027505ar
Résumé de l'article

Malgré L'attention considérable des politiques dans ce domaine, le nombre de suicides dans les prisons d'Angleterre et du pays de Galles continue de poser un problème. Suite à la prolifération des études entreprises, d'importants progrès ont été réalisés. Nous allons ici revoir les conclusions clés de différentes études menées dans plusieurs pays au cours des dix dernières années. De plus en plus, le suicide en prison est considéré comme un problème de vulnérabilité et d'adaptation, et l'on croit qu'en améliorant les politiques et les relations on pourra offrir aux prisonniers ce soutien dont ils ont un si grand besoin. Les liens entre la capacité à résoudre des problèmes, l'adaptation, le soutien social et le comportement suicidaire en prison sont évidents ; le présent article soutient donc qu'il faut aborder le suicide dans le cadre des relations sociales interpersonnelles pour arriver à le comprendre. La recherche devrait s'appuyer sur la littérature sociologique facilement disponible et qui insiste sur l'importance des réseaux sociaux au sein de la communauté carcérale. Les détenus qui constituent un risque de suicide sont souvent les plus démunis quand il s'agit d'obtenir du soutien social ou de réagir aux rares sources de soutien disponible. Parallèlement aux méthodes psychologiques et aux tests psychométriques, la recherche devrait s'appuyer sur l'ethnographie et sur les modèles qualitatifs, ainsi que sur l'examen systématique du tissu complexe des relations existantes dans la communauté carcérale. Si cet article sert de tremplin à de plus amples discussions et qu'il génère davantage de recherche, son but aura été atteint.
Ce document est protégé par la loi sur le droit d'auteur. L’utilisation des services d'Érudit (y compris la reproduction) est assujettie à sa politique d'utilisation que vous pouvez consulter en ligne.

https://apropos.erudit.org/fr/usagers/politique-dutilisation/ 


\title{
Suicide et tentatives de suicide en prison : vulnérabilité, ostracisme et soutien social ${ }^{1}$
}

\author{
Joel Harvey ${ }^{2}$ \\ Combridge Institute of Criminology \\ United Kingdom \\ jarh3@com.oc.uk
}

\author{
Alison Liebling \\ Cambridge Institute of Criminology \\ United Kingdom \\ al115@com.ac.uk
}

Sommaire - Malgré l'attention considérable des politiques dans ce domaine, le nombre de suicides dans les prisons d'Angleterre et du pays de Galles continue de poser un problème. Suite à la prolifération des êtudes entreprises, d'importants progrès ont été réalisés. Nous allons ici revoir les conclusions clés de différentes études menées dans plusieurs pays au cours des dix dernières années. De plus en plus, le suicide en prison est considéré comme un problème de vulnérabilité et d'adaptation, et l'on croit qu'en améliorant les politiques et les relations on pourra offrir aux prisonniers ce soutien dont ils ont un si grand besoin. Les liens entre la capacité à résoudre des problèmes, l'adaptation, le soutien social et le comportement suicidaire en prison sont évidents ; le présent article soutient donc qu'il faut aborder le suicide dans le cadre des relations sociales interpersonnelles pour arriver à le comprendre. La recherche devrait s'appuyer sur la littérature sociologique facilement disponible et qui insiste sur l'importance des réseaux sociaux au sein de la communauté carcérale. Les détenus qui constituent un risque de suicide sont souvent les plus démunis quand il s'agit d'obtenir du soutien

1. Texte traduit par André Bourbonnière.

2. Joel Harvey poursuit un doctorat au Cambridge Institute of Criminology. Alison Liebling est adjointe de recherche principale et directrice du Prisons Research Centre. Les auteurs tiennent à remercier Helen Griffiths et Joy Anderson pour leur travail de secrétariat et leur appui. Ils tiennent également à remercier le ESRC pour les deux bourses qui ont permis la recherche à la base du présent article. Ils tiennent aussi à exprimer leur gratitude à la Suicide Awareness Support Unit, source de l'information sur les taux de suicide utilisés ici. 
social ou de réagir aux rares sources de soutien disponible. Parallèlement aux méthodes psychologiques et aux tests psychométriques, la recherche devrait s'appuyer sur l'ethnographie et sur les modèles qualitatifs, ainsi que sur l'examen systématique du tissu complexe des relations existantes dans la communauté carcérale. Si cet article sert de tremplin à de plus amples discussions et qu'il génère davantage de recherche, son but aura été atteint.

summary - Prison suicides in England and Wales continue and present a problem despite considerable policy attention. Research has proliferated and some important advances have been made. Key findings from studies carried out over the last decade in several countries are reviewed. Prison suicide is increasingly seen as a problem of vulnerability and coping. Improvements to regimes and relationships can offer much needed support. There are clear links between problem-solving, coping, social support and suicidai behaviour in prison. This paper argues that prison suicide needs to be understood within a framework of interpersonal social relationships. Research should draw on readily available sociological literature, which highlights the significance of social networks in the prison community. Those at risk of suicide may be least likely to have, be able to summon, or respond to scarce sources of social support. Ethnographic and other qualitative modes of research should be used to supplement psychological methods and the use of psychometric tests. The complex web of relationships that constitute the prison community should be systematically examined. The aim of the paper is to serve as a springboard for further discussion and for the generation of further research.

- Je me sentais comme exclu, de trop, à part de mon frère et de mes sours. J'avais l'impression d'être rejeté. Non, je n'en ai jamais parlé. Le problème était simplement toujours là. Je crois que je voulais surtout me venger d'eux, mais pas pour attirer l'attention; ils ne l'ont jamais su.

Les lettres font du bien, mais pas les visites. Ça bouleverse. Parfois, tu te sens complètement dégoûté. Tout dépend comment ça se passe.

Je veux changer ma façon de vivre.

- Comment allez-vous y arriver?

- Je ne sais pas.

\section{Introduction}

En institution comme dans la communauté, suicide et automutilation sont souvent perçus comme « des actes irréfléchis » méritant parfois une punition (Liebling et Ward 1995 ; Bailey, 1998). Mais cette position est insoutenable, car ces actes sont l'expression éloquente d'une insupportable souffrance. À l'extérieur des murs, le suicide est plutôt un acte privé et insondable "préparé dans le silence du cœur" (Camus, 1955). En prison, ce geste devient public et requiert enquête et réponse de l'insti- 
tution. Depuis 1994, en Angleterre et au pays de Galles, surgit une prolifération de stratégies visant à prévenir le suicide dans les prisons ; les progrès sont notables, bien que le problème persiste. Pour l'année 1998 , dans les prisons de ces deux pays, on a relevé 82 morts autoinfligées, soit un taux annuel de 125 par 100000 détenus pqm (population quotidienne moyenne). En 1999, ce nombre passait à 91 , soit 140 par 100000 , alors qu'il tombait à 81 en 2000 , un taux de 127 par 100000 (Safer Custody Group, 2001). Cette situation révèle donc la nécessité de poursuivre les efforts en matière de politique et de recherche dans ce domaine. Nous soutenons que l'étude du suicide en prison devrait se construire sur une compréhension mieux intégrée des connaissances disponibles sur l'expérience de la détention. Ces connaissances incluent toute la littérature en sociologie et en psychologie, tout comme les modèles de comportements autant sociaux que psychologiques.

Depuis les dernières années, l'étude du suicide en prison se fait à l'échelle internationale et les progrès réalisés sont importants. Des recherches ont été menées en Australie (Dalton, 1999 ; Dear et al., 1998 ; 2000 ; sous presse ; Dear 2000), au Canada (Green et al., 1993 ; Wichmann et al., 2000), en Italie (Tatarelli et al., 1999), en Écosse (Bogue et Power, 1995 ; Biggam et Power, 1999), en Angleterre et au pays de Galles (Liebling, 1992 ; Liebling et Krarup, 1993 ; Inch et al., 1995 ; Crighton et Towl, 1997 ; Towl, 1999; White et al., 1999), en Nouvelle-Zélande (Skegg et Cox, 1992), en Suisse (Schaller et al., 1996), en Finlande (Joukamma, 1997 ; 1998), aux États-Unis (Bonner et Rich, 1990 ; Ivanoff et Jang, 1991 ; Bonner, 1992 ; Ivanoff, 1992 ; Lester et Danto, 1993 ; Smyth et al., 1994 ; Du Rand et al., 1995 ; Hayes et al., 1995 ; Ivanoff et al., 1992 ; 1996 ; Fulwiler 1997), en France (Bourgoin, 1993 ; Benezech, 1999) et en Autriche (Fruhwald et al., 2000). Ces études englobent l'identification des taux et des facteurs de risque associés aux suicides, l'examen des facteurs de risque et des motifs derrière l'automutilation et les tentatives de suicide, et l'analyse spécifique du rôle de certaines variables particulières en relation avec l'automutilation /la tentative de suicide et l'adaptation. Dans l'ensemble, ces études s'attachent uniquement au comportement suicidaire chez les hommes; ce n'est que dernièrement que les chercheurs ont commencé à considérer officiellement la dimension sociale autant qu'individuelle du risque de suicide. La majeure partie du travail était de nature quantitative, bien qu'on remarque un intérêt croissant pour les études qualitatives et prospectives. 


\section{Historique}

En janvier 1999, l'Organisation mondiale de la santé (OMS-Europe) mettait sur pied un groupe de travail chargé de revoir les politiques relatives au suicide dans les prisons et d'apporter, dans toute la mesure du possible, des améliorations. En juillet de la même année, le Parlement du Royaume-Uni recevait un livre blanc intitulé Saving Lives: Our Healthier Nation ; entre autres, cette démarche visait à « réduire le taux des mortalités par le suicide ou de cause indéterminée d'au moins un cinquième d'ici à 2010 - sauvant ainsi près de 4000 vies " (House of Parliament, 1999). En avril 1994, le Service des prisons d'Angleterre et du pays de Galles introduisait une nouvelle stratégie, Caring for the Suicidal in Custody, qui allait marquer une nouvelle tendance dans la prévention du suicide dans les prisons. Le suicide n'était plus considéré comme un problème relevant uniquement du personnel médical ; il devenait la responsabilité de tout le personnel de la communauté carcérale. Dans cette optique, l'organisation The Samaritans forma des Listeners schemes, c'està-dire des prisonniers prêtant une oreille attentive pour aider leurs codétenus. Au cœur même de cette stratégie se trouve une approche de communauté carcérale «favorisant les relations de soutien dans l'idée qu'on peut amener une personne suicidaire à s'en sortir pourvu qu'elle accepte l'aide offerte » (Home Office, $1999: 46$ ).

Cette notion de « responsabilité collective" a été portée plus avant lors d'une récente revue thématique menée par l'inspecteur en chef des prisons de Sa Majesté pour l'Angleterre et le pays de Galles. Ce rapport faisait valoir que le « suicide est. '? affaire de tous » appuyant sur l'objectif plus large de développer des "prisons saines", soit des institutions "dans lesquelles prisonniers et personnel sont capables de vivre et de travailler ensemble de façon à favoriser leur bien-être " (Home Office, 1999 : 59). Cette approche holistique insiste sur le fait que l'on ne pourra s'attaquer efficacement au problème du suicide en prison tant que les efforts se limiteront au problème de l'individu ; l'attention doit également porter sur les régimes institutionnels et les relations en général. Cette pensée correspond assez bien aux conclusions de la recherche (par exemple, Backett, 1988 ; Liebling, 1992 ; Biggam et Power, 1997).

Plus récemment, en avril 2001, le Safer Custody Group du Service des prisons de Sa Majesté a proposé une stratégie triennale par laquelle " on allait établir dans les prisons une approche dynamique complète encourageant une culture de soutien basée sur des relations saines entre 
personnel et prisonniers, un régime constructif et un environnement physiquement sûr. Les prisonniers à risque élevé seront mieux identifiés et feront l'objet de dispositions particulières " (Safer Custody Group, 2001 - Notre traduction).

Le présent article soutient que l'on doit aborder le suicide dans les prisons selon un cadre de relations sociales interpersonnelles. S'il sert de tremplin à de plus amples discussions et qu'il génère davantage de recherche, nous aurons atteint notre but.

\section{Cheminement vers le suicide : vulnérabilité et adaptation à la prison}

Liebling (1992) a mis en évidence l'importance de l'adaptation au milieu carcéral dans la manifestation des comportements suicidaires. Le modèle " vulnérabilité » a surgi d'une recherche qui s'écartait des études précédentes en ce qu'elle comportait des entrevues avec des prisonniers, jeunes ou adultes, hommes ou femmes, qui avaient fait une tentative de suicide pour les comparer à d'autres détenu(e)s n'ayant pas attenté à leur vie. Les études précédentes s'étaient concentrées sur les suicides en cherchant à identifier les facteurs de risque précurseurs et en tentant d'établir un profil de détenu suicidaire à partir de documents officiels et des rapports au dossier (Danto, 1973 ; Burtch et Ericson, 1979 ; Topp, 1979 ; Backett, 1987 ; Dooley, 1990). Ces rapports, a-t-on soutenu, ne fournissaient qu'une image incomplète en raison des partis pris, des inexactitudes et des lacunes qu'ils contenaient. Plusieurs des études précédentes avaient omis d'inclure un groupe témoin sans lequel il est impossible de déterminer si les facteurs relevés établissent une différence entre le groupe suicidaire et le reste de la population carcérale. Nombre des facteurs de risque associés au suicide se retrouvent dans l'ensemble de la population carcérale.

La recherche a révélé que ceux qui avaient tenté de se suicider possédaient moins de qualifications, étaient des fugueurs fréquents, avaient été plus souvent impliqués dans la violence en milieu scolaire (souvent victimes de brutalité) et étaient plus susceptibles d'être sous la surveillance des autorités locales (ceci faisait probablement suite à des problèmes de famille ou de comportement plutôt qu'à un comportement délictuel). Ils étaient également plus susceptibles d'avoir été traités en psychiatrie, en milieu hospitalier autant qu'en clinique externe, plus susceptibles de s'être automutilés avant d'être en détention préventive et 
plus susceptibles de présenter des problèmes d'alcool et de drogue. Bien que les deux groupes provenaient de milieux familiaux instables, le groupe suicidaire était caractérisé par de plus hauts taux de violence familiale et de pathologie psychiatrique. Les variables de la justice criminelle révélèrent peu de différences entre ceux qui avaient tenté de se suicider et ceux qui n'avaient pas tenté de le faire, si ce n'est que les premiers présentaient des antécédents de condamnations légèrement supérieurs au groupe témoin.

La plus grande différence résidait dans le mode de vie de chacun des groupes en prison. L'exploration des relations sociales interpersonnelles en prison révéla que ceux qui avaient tenté de se suicider étaient moins susceptibles de qualifier « d'amis » leurs codétenus, étaient plus susceptibles de les avoir connus en prison plutôt qu'à l'extérieur, avaient plus de problèmes avec les autres détenus et moins de contacts avec le service de probation. Sans compter que, si contact il y avait, celui-ci était considéré comme moins utile. En termes de relations sociales interpersonnelles à l'extérieur de la prison, ceux qui avaient tenté de se suicider étaient moins susceptibles de recevoir des visiteurs, écrivaient moins de lettres, étaient moins en rapport avec des gens et s'ennuyaient de certaines personnes plus que les autres détenus. Les détenus suicidaires passaient plus de temps oisifs en cellule, étaient plus susceptibles d'être rébarbatifs à l'exercice physique, s'ennuyaient plus, rêvassaient plus, souffraient plus d'insomnie, se plaignaient plus du système disciplinaire et éprouvaient plus de difficultés avec l'incarcération.

Le suicide en prison serait « en grande partie un problème d'adaptation" (Liebling, 1992: 236). L'auteur a trouvé que les " détenus qui avaient le moins d'occasions de se distraire » (quelle que soit la raison, parfois par leur faute) étaient les " moins capables de faire face à l'isolement et à l'ennui de la réclusion en cellule sur une période prolongée " (Liebling, 1992 : 144). Selon cette auteure, "l'emprisonnement est imposé à des groupes vulnérables » et "supposés subir une expérience où les exigences peuvent dépasser les ressources disponibles. Résultat : souffrance. Conséquence : taux excessifs de suicides» (Ibid. : 184).

À partir de cette recherche et d'une reproduction de l'étude auprès des prisonniers adultes, on a établi un modèle théorique du cheminement vers le suicide, basé sur la notion de vulnérabilité et d'adaptation (Liebling, 1999), et révisé ici (voir schéma 1). Selon ce modèle, les pensées et le comportement suicidaires suivent un continuum et englobent la vulnérabilité, le stress de l'emprisonnement et des déclencheurs 
situationnels, de façon interactive et dynamique. Les prisonniers peuvent arriver déjà empreints de vulnérabilité marquée, d'où leur difficulté d'adaptation au stress de l'emprisonnement et aux déclencheurs situationnels (mauvaises nouvelles, fin d'une relation et ainsi de suite). Certains prisonniers étaient par ailleurs moins vulnérables, mais ils étaient confrontés à des difficultés situationnelles graves en prison. Le niveau de vulnérabilité d'un individu dépend alors des ressources internes cognitives et émotionnelles, et des ressources externes dont il dispose ou non pour gérer le stress de l'incarcération. En général, ceux qui ne disposent pas de ressources cognitives et émotionnelles pour gérer ce stress sont plus prédisposés aux problèmes d'ajustement pouvant mener à une détresse psychologique. Ces détenus se retrouvent souvent dans les pires situations en prison. En effet, « ces détenus moins débrouillards sont exposés aux plus sérieux agents provocateurs ». Liebling soutient que « c'est le cumul de la sensation d'impuissance, de leurs antécédents et de leur situation actuelle, plus le fait qu'ils n'arrivent à trouver ni solution à cette situation ni personnes pouvant les aider à générer des solutions, qui poussent les prisonniers au suicide » (1999: 328). Les déclencheurs situationnels pourraient être " déterminants dans une tentative de suicide à différents paliers, dépendant à la fois de la vulnérabilité du prisonnier et de son niveau de stress » (Ibid. : 329). On a relié la difficulté d'identifier le risque de suicide au moment de l'arrivée en détention à la nature dynamique plutôt que statique du risque. Parmi les agents de protection, on compte plusieurs aspects de soutien social, y compris les visites et le contact avec la famille, une occupation constructive en prison, l'appui des autres prisonniers et du personnel, et le fait d'avoir de l'espoir et des projets d'avenir. Tous ces éléments constituent un bon modèle de vie en captivité malgré la présence du risque de suicide.

D'autres études se sont penchées sur le concept d'adaptation. En Australie-Occidentale, une étude menée par Dear et al. (sous presse) s'est attachée aux différences entre les détenus masculins qui s'étaient automutilés et les autres. Les auteurs cherchaient à identifier les facteurs clés reliés au milieu social, au fonctionnement psychologique et à la vulnétabilité psychosociale établissant les différences entre les automutilateurs et un groupe de comparaison apparié. On interviewa 71 individus qui s'étaient automutilés au cours des trois jours précédents, et 71 détenus du groupe témoin. Les questions portaient sur leurs antécédents d'automutilation et sur leur incarcération présente (en particulier, sur les 
événements porteurs de stress et sur leurs relations sociales). Les détenus furent aussi soumis à des tests psychologiques pour déterminer leur potentiel de suicide et leur détresse psychologique. Les détenus qui s'étaient automutilés ont démontré plus de vulnérabilité, de détresse ou de désordre, leurs relations étaient plus faibles, ils étaient plus susceptibles de menaces ou d'intimidation de la part des autres prisonniers, ils nommaient moins de ressources sociales, étaient moins susceptibles de considérer suffisant leur niveau actuel de soutien social, et leur adaptation était plus faible. Sur le plan des antécédents, toutefois, les différences n'étaient pas très grandes. Bien que les antécédents d'automutilation étaient plus fréquents au sein du groupe des automutilés, près d'un tiers du groupe témoin avait connu la même expérience. Le fait que les deux groupes n'étaient pas réciproquement exclusifs limite la portée des conclusions de cette étude.

Biggam et Power (1999a) ont pour leur part mené une étude en Écosse, comparant la capacité de résolution de problèmes et la détresse psychologique chez les détenus suicidaires de sexe masculin (ceux dits "sous stricte supervision suicidaire », SSS), les victimes de harcèlement, les détenus sous protection pour leur propre sécurité et un groupe témoin. Pour mesurer la détresse psychologique, les chercheurs utilisèrent les tests suivants : Hospital Anxiety and Depression Scale (HADS), Profile of Mood Scale (POMS) et Beck's Hopelessness Scale (BHS). Pour mesurer sa capacité à résoudre ses problèmes, chaque détenu a été soumis au test Means-End Problem Solving (MEPS), dans lequel l'individu doit apporter des solutions aux problèmes interpersonnels hypothétiques qui lui sont présentés. Les auteurs ont fait le compte des solutions "pertinentes" et des «non pertinentes» fournies, de même que des réponses «actives» et "passives», pour en arriver aux conclusions suivantes : en ce qui concerne l'état affectif de chacun, il semble y avoir une hiérarchie dans la détresse, c'est-à-dire que les détenus SSS démontrent le niveau le plus élevé de détresse (souvent à un niveau requérant une intervention clinique), suivis des victimes de harcèlement, puis des détenus placés sous protection. Le plus faible niveau de détresse se retrouve chez le groupe témoin (Biggam et Power, 1999:211). Les auteurs ont trouvé, pour l'ensemble de l'échantillon, une corrélation entre détresse psychologique accrue et déficience dans la capacité à résoudre des problèmes. Comme cette étude est intersectionnelle et corrélationnelle de nature, il n'est pas possible de déterminer la directionnalité. Mais les auteurs notent qu'il est " plausible que ces deux 
conditions soient antérieures à l'appartenance à un groupe particulier, bien que les influences situationnelles du statut du détenu soient susceptibles d'accroître la détresse " (Biggam et Power, 1999a : 212). Le fait que cette étude ne mesure pas le risque suicidaire constitue une faille importante. Les détenus du groupe suicidaire ont été les 25 premiers cas amenés en consultation sous stricte supervision suicidaire. Il demeure possible que les détenus des trois autres groupes aient également fait preuve de pensées ou de comportements suicidaires, antérieurement et/ ou présentement. Encore une fois donc, il reste possible que ces groupes ne soient pas mutuellement exclusifs. On a évalué la capacité à résoudre des problèmes en utilisant le test MEPS, qui consiste à trouver une solution à un problème. En prison, une adaptation réussie pourrait ne pas être tellement une question de « résolution de problème » que de gestion des «problèmes » et des «émotions".

Les carences dans la capacité à résoudre des problèmes ont joué un rôle important dans la recherche sur le suicide dans la communauté en général (Schotte et Clum, 1982; 1987 ; Schotte et al., 1990), et, plus récemment, dans le cadre de l'établissement des peines (Ivanoff et al., 1992 ; Biggam et Power, 1999b). Le débat persiste quant à savoir si ces carences doivent être interprétées comme un phénomène relié à un trait de caráctère ou à un état. Biggam et Power (1999b) ont mené une étude sur le débat état-trait relatif à ces carences auprès de 61 jeunes délinquants écossais de sexe masculin, âgés de 16 à 21 ans. Leur étude s'apparentait à une étude antérieure menée par Ivanoff et al. (1992) auprès de 93 détenus masculins aux États-Unis. Biggam et Power comparèrent trois groupes : les individus ayant des antécédents de parasuicide (conduite suicidaire non fatale) et étant considérés comme suicidaires au moment de l'étude, ceux qui avaient des antécédents de parasuicide mais qui n'étaient pas suicidaires à ce moment-là, et ceux qui n'avaient pas d'antécédents et n'étaient pas du tout suicidaires à ce moment-là. Les détenus classifiés comme suicidaires avaient tenté de s'automutiler au cours des 72 heures précédentes et, pour cette raison, se trouvaient sous SSS. Les tests HADS et BHS avaient servi à mesurer la détresse psychologique; on avait encore une fois utilisé le MEPS pour mesurer la capacité à résoudre des problèmes.

Afin de tester les effets d'état, on examina seulement les détenus avec des antécédents de parasuicide, en les comparant entre eux selon qu'ils étaient ou non suicidaires au moment précis de l'étude. Les auteurs se rendirent compte que la détresse psychologique était plus grande en 
termes de niveau élevé de dépression chez ceux qui étaient suicidaires à ce moment-là. Le désespoir était cependant également élevé chez les deux groupes. Côté capacité à résoudre des problèmes, les détenus activement suicidaires démontraient de plus grandes carences à cet effet. Cet énoncé est à l'encontre de l'étude menée par Ivanoff $e t$ al. (1992b) qui n'avait révélé aucune différence entre les deux groupes. Pour tester les effets " trait ", on se limita aux détenus non activement suicidaires, en les comparant entre eux selon qu'ils possédaient ou non des antécédents de parasuicide. Contrairement à Ivanoff et al. (1992b), les auteurs trouvèrent que la détresse psychologique était plus grande chez ceux qui avaient des antécédents de parasuicide comparativement à ceux qui étaient sans antécédents. En ce qui concerne toutefois les carences dans la capacité à résoudre des problèmes, leurs conclusions confirment celles de Ivanoff et al. (1992b), n'établissant aucune différence entre les deux groupes. Rassemblant ces résultats, Biggam et Power (1999b) suggèrent que «bien que la capacité à résoudre des problèmes ne soit pas un phénomène de trait de caractère, elle pourrait être un état corollaire au risque suicidaire " et que " la détresse psychologique est à la fois un trait et un état indicateurs de comportement parasuicidaire " (Biggam et Power, 1999b : 37).

En 1997, Haines et Williams ont mené une dernière étude portaṇt sur l'adaptation et la capacité à résoudre des problèmes des gens qui s'automutilent. Ils soutiennent que ces derniers obtiennent des résultats inférieurs quant à l'estime de soi et au contrôle personnel, possèdent moins de ressources sociales et ont recours à plus de stratégies d'évitement des problèmes et de retrait émotionnel, démontrant des ressources cognitives réduites. Les auteurs notent que, dans certaines études, l'automutilation est considérée comme une stratégie d'adaptation, quoi qu'il s'agisse d'une stratégie faible.

Des études sur le stress et l'adaptation dans la communauté suggèrent que les styles d'évaluation et d'adaptation varient (Lazarus, 1966 ; Lazarus et Folkman, 1984 ; Lazarus, 2000). On parle d'adaptation axée sur le problème «lorsqu'une personne obtient de l'information qui va guider ses actions et qu'elle mobilise ses actions dans le but de changer la réalité de la relation troublée personne-environnement " (Lazarus, 2000 : 204). Par contre, l'adaptation axée sur l'émotion implique "l'ajustement des émotions liées à la situation de stress " (Lazarus $2000: 204$ ). On soutient que l'adaptation axée sur l'émotion surgit surtout lorsqu'on ne peut changer l'environnement, alors que l'adapta- 
tion axée sur le problème tente d'apporter des changements dans l'environnement même. Au sein de la prison, il est permis de croire que l'adaptation axée sur l'émotion revêt plus d'importance pour permettre au détenu de survivre psychologiquement. La situation qui prévaut présentement risque peu de changer en profondeur ; par conséquent, ceux qui sont en mesure de gérer leur détresse émotionnelle ont plus de chance de bien gérer leur vie. Gallo et Ruggiero (1991) soutiennent qu'en prison, "l'effort pour maintenir sous contrôle la détresse même " engendre une certaine quantité de détresse (p. 323). Toutefois, Lazarus (2000) remarque que les deux types d'adaptation (axée sur l'émotion et axée sur le problème) se complètent réciproquement et qu'ils sont nécessaires pour s'adapter efficacement. Negy et al. (1997) se sont intéressés à la relation entre adaptation et ajustement auprès de 153 femmes détenues dans une prison à sécurité minimale; ils en concluent que les deux stratégies, axée sur l'émotion et axée sur le problème, étaient en corrélation positive avec l'ajustement psychologique.

Inch et al. (1995) ont mené des entrevues semi-structurées avec 25 jeunes délinquants masculins (âgés de 16 à 21 ans) qui s'étaient automutilés, et 25 autres sujets témoins. Selon leur définition, l'automutilation consiste en " toute tentative de s'infliger une blessure ", avec des incidents survenant habituellement dans l'espace d'une semaine. Ils n'ont trouvé aucune différence significative en ce qui concerne l'expérience de travail, le temps passé sous la surveillance des autorités locales ou la scolarité. La différence était cependant importante en ce qui a trait aux antécédents d'automutilation ( $70 \%$ pour le premier groupe, $12 \%$ pour le groupe témoin) et les antécédents familiaux d'automutilation ( $24 \%$ et $18 \%$ respectivement). On a également relevé des différences par rapport à la détresse psychologique, les détenus s'étant automutilés démontrant " des niveaux accrus de détresse subjective [...] se manifestant sous forme d'anxiété, de symptômes de dépression et d'absence d'espoir face à l'avenir " (Inch et al., 1995: 167). Bien qu'on ait trouvé des différences quant au nombre et aux types « de facteurs de risque » (comme des antécédents d'automutilation), les auteurs préviennent que l'approche d'identification du tisque peut mener à des « excès de bureaucratie, une attitude défensive et des réactions inappropriées », et qu'il faut s'attaquer "à la culture et aux attitudes sous-jacentes" (p. 168). Suite à ces entrevues, les auteurs ont découvert que le harcèlement, ainsi que les difficultés familiales et de relations, étaient les raisons le plus souvent évoquées en rapport avec l'automutilation (respective- 
ment $44 \%$ et $20 \%$ des réponses). Les difficultés familiales et de relations incluaient " la perte de contact, le reniement, la fin d'une relation et les inquiétudes relativement aux réactions de la famille face à l'emprisonnement " (Inch et al., 1995 : 167). De plus, $32 \%$ de ces détenus ont déclaré avoir de la difficulté à s'adapter et être accablés par le fait d'être " enfermés » et "bouclés pour la nuit ». Inch et al. ont conclu que "le fil commun reliant presque tous ces actes d'automutilation dans notre étude était ce désir désespéré de fuir une situation devenue intolérable et qui avait écrasé les mécanismes d'adaptation des individus en question » (Inch et al., 1995 : 168).

Finalement, dans une revue des études pertinentes, le Service correctionnel du Canada s'est penché sur les différences entre les détenus ayant tenté de se suicider et les autres (Wichmann et al., 2000). Les deux principaux objectifs de cette étude consistaient à identifier les variables importantes pour l'évaluation du risque et à tester la validité de prédiction de "l'échelle du potentiel de suicide », utilisée à l'admission des détenus. Les données ont été recueillies dans les dossiers d'incidents de 731 prisonniers masculins qui avaient tenté de se suicider comparativement à 731 prisonniers dont les dossiers ne rapportaient pas un tel comportement. Les analyses ont tévélé que ceux qui avaient tenté de se suicider étaient le plus souvent de race blanche, plus jeunes (moins de 30 ans), non mariés, coupables d'homicides et de vols, moins susceptibles d'avoir commis des délits d'ordre sexuel ou reliés à la drogue, plus susceptibles de purger une sentence à vie et plus susceptibles d'être placés dans une institution à sécurité maximale lors de l'admission. Les résultats quant au statut marital variaient cependant légèrement en fonction de l'âge. Les analyses portaient sur le fonctionnement psychologique de 76 détenus ayant tenté de se suicider et un groupe témoin de 76 appariés, selon l'âge à l'admission, le type de délit et la durée de la sentence. On isolait ainsi ces caractéristiques statiques dans le but de faire ressortir la contribution des facteurs de risque dynamiques. Les auteurs se sont rendus compte que les détenus qui avaient tenté de se suicider démontraient plus de problèmes extériorisés ou intériorisés, avaient des antécédents psychiatriques plus considérables, provenaient de familles plus dysfonctionnelles, et avaient des dossiers disciplinaires contenant un plus grand nombre de rapports d'incidents. Ils en vinrent à la conclusion que " le fonctionnement psychologique des délinquants et la qualité du soutien familial et social qu'ils reçoivent, même lorsque évalués à l'admission dans une institution fédérale, différencient ceux 
qui tentent de se suicider des autres " (Wichmann et al., $2000: 23$ ). On en vint également à la conclusion que ceux qui allaient faire une tentative de suicide avaient un passé criminel plus chargé que les autres. À partir de deux éléments mesurés sur l'échelle du potentiel de suicide (tentative antérieure de suicide et intervention psychologique et/ou psychiatrique) ainsi que trois autres variables (problèmes de discipline, incidents impliquant de la contrebande et condamnations antérieures à l'âge adulte), " ont permis de prédire la majorité des tentatives de suicide par les délinquants inscrits dans l'étude (92\%) - même si ces tentatives sont survenues ultérieurement au cours de la sentence " (Wichmann et al, 2000 : vii). Cette étude se base principalement sur des variables actuarielles d'évaluation du risque et sur les caractéristiques individuelles; elle ne contient aucune donnée recueillie au cours d'entrevues avec les prisonniers.

De toute évidence, il existe un rapport entre la résolution des problèmes, l'adaptation, le soutien social et le comportement suicidaire en prison. En effet, « depuis les trente dernières années, tant dans les écrits professionnels que dans les autres types d'écrits, on s'accorde sur le potentiel du soutien social pour réduire le stress et améliorer l'adaptation » (Underwood, 2000 : 367). Underwood note que les études se sont concentrées sur la différenciation des sources de soutien social, à savoir : qu'est-ce qui est réellement offert en termes de soutien et si le soutien agit de façon unidirectionnelle ou réciproque. Elle déclare que " la précision conceptuelle est au cœur même de la question, et qu'il est essentiel de tester systématiquement les éléments explicités et leur influence sur les autres variables dans un contexte de stress et d'adaptation" (Underwood, $2000: 369$ ). Comme d'autres études ont réussi à le démontrer, l'adaptation et le soutien social peuvent être modifiés à un niveau individuel par d'autres conditions, tels l'impuissance acquise, le désespoir et le lieu de contrôle (Jack, 1992 ; Liebling et Krarup, 1993).

\section{Vulnérabilité et soutien social}

Évidemment, on a également fait des recherches portant sur le lien entre le suicide et le soutien social au sein de la communauté en général. On peut attribuer l'origine de ces idées à Durkheim (1897), qui soutenait que l'intégration sociale était d'une importance capitale pour expliquer les taux relativement stables de suicide au sein de différentes sociétés. 
Les sociétés où les taux de suicide étaient plus faibles étaient considérées plus unies ou religieuses, alors que les taux plus élevés se retrouvaient dans les sociétés moins intégrées. Durkheim a baptisé suicide " égoïste " le suicide découlant d'un manque d'intégration. Il soutenait également que le suicide pouvait provenir d'une intégration excessive, d'où l'étiquette de suicide « altruiste». Le soutien social à un niveau individuel — quant à son apport, sa forme et son efficacité — prend clairement une importante dimension dans le cadre général de «l'intégration sociale ".

Dans leur recension sur le sujet, Westefeld et al. (2000) concluent que "l'absence de soutien social est pertinente pour prédire le suicide. Un soutien social accru réduit le risque chez la personne suicidaire et accroît la probabilité d'obtenir du soutien social après une tentative de suicide " (Westefeld et al., 2000 : 452). Morano et al. (1993) ont trouvé que le niveau de soutien social disponible et la perte récente d'une personne aimée différenciaient les adolescents qui allaient tenter de se suicider et les autres, ces deux groupes étant appariés au niveau de la dépression.

La littérature relative aux prisons traite peu du rôle que joue le soutien social. Selon Biggam et Power (1997), « on pourrait considérer le soutien social comme une importante variable psychologique et sociale, qui contribue à l'ajustement en prison et à l'amélioration du stress " (p. 214). Les auteurs sont d'avis que le soutien social agit comme une " aide à l'adaptation ». Dans leur étude qui portait sur 125 jeunes délinquants masculins en Écosse, ces derniers identifiaient les relations importantes sous l'échelle "Significant Others Scale ", puis ils devaient classer chacune de ces relations en termes de soutien " réel " par rapport au soutien "idéal ". Ils se sont rendus compte que les " détenus affichant de la détresse psychologique souhaitaient plus de soutien de la part de leurs codétenus » (pp. 225-226) et plus de soutien émotionnel et pratique de la part des agents. En effet, " les détenus vulnérables expriment leur désir d'une relation plus " thérapeutique » avec le personnel » (Biggam et Power, $1997: 226$; voir aussi Liebling et Price, 1999). La perception d'un manque à ce niveau « constituait le principal facteur de prédiction de l'anxiété, de la dépression et du désespoir » (p. 226). Leur étude représente une contribution importante d'un domaine sous-exploité.

Bien que de plus en plus la recherche s'accorde pour dire que le soutien social est d'importance capitale quand on analyse le comportement suicidaire (voir par exemple Eskin, 1995), peu d'études ont tenté d'explorer de façon systématique les processus impliqués, comme le soutiennent Biggam et Power (1997). Bonnet (2000) déclare qu'il est encore 
"difficile d'établir si une perturbation au niveau des contacts et du soutien social exerce la même influence chez les détenus qui se suicident que chez les autres " (p. 371). Bien des questions restent sans réponse. Quel est l'impact du manque de soutien social sur la manifestation de comportements suicidaires? Quel rôle exerce le fait d'avoir et d'être capable de former des relations sociales dans le processus d'adaptation et l'expérience de la vie en prison ? En quoi consiste le soutien social, de même que le manque de ce soutien? À qui les prisonniers peuvent-ils faire confiance? En quoi consiste le soutien émotionnel ? À un macroniveau, il peut être important de différencier relations et interactions, ainsi que le support qui en découle respectivement (van Aken $e t a l$,, 1999). La recherche récente sur les relations personnel-prisonniers et certains ouvrages sociologiques classiques sur les prisons établissent ces distinctions importantes entre « interactions » et « relations » (Liebling et Price, 1999 ; voir aussi Ben-David et Silfen, 1994) et entre " amitié » et "solidarité " (Sykes, 1958). Une évaluation nationale des mesures d'encouragement et des privilèges mérités s'est penchée sur les niveaux de confiance; les constatations permettent de penser que les détenus font plus confiance au personnel qu'aux autres détenus (Liebling et al., 1997). L'étude des réseaux sociaux en prison compose une partie importante de la tradition sociologique classique (voir Clemmer, 1940 ; Sykes, 1958 ; Irwin et Cressey, 1962 ; Mathiesen, 1965), mais ces études ne font pas la différence entre les individus vulnérables et les autres groupes de détenus, pas plus qu'elles ne se sont penchées sur le risque de suicide. Il existe des règles régissant la sociabilité et la marginalisation en prison (Gallo et Ruggiero, 1991 ; et voir plus bas) et le paradoxe des relations personnel-prisonniers repose précisément sur cette tension (" courant à deux sens") entre soutien et domination (ibidem : 276 ; Shapira et Navon, 1985 ; Liebling, 2000). Cette précieuse documentation sociologique n'a cependant pas servi à l'étude de l'adaptation, du soutien social et de la vulnérabilité en prison ${ }^{3}$.

Bien que les individus suicidaires soient, en partant, moins susceptibles de disposer de ressources informelles de soutien social, ils peuvent également être moins portés à utiliser les ressources existantes

3. L'ouvrage Psychological Survival de Cohen et Taylor, qui explore l'amitié, le temps, l'intimité et la crainte de détérioration parmi les prisonniers des établissements à sécurité maximale, donne probablement l'approximation la plus rapprochée pour ce genre d'application, mais cette étude n'était qu'exploratoire et " officieuse " (voir Cohen et Taylor, 1972). 
auxquelles ils pourraient avoir recours. Cette situation peut aussi s'appliquer à leur degré d'utilisation des Listener Schemes ${ }^{4}$. On peut se demander si un détenu suicidaire fera l'effort de demander de l'aide. Carlton et Deane (2000) ont étudié l'impact des idéations suicidaires des adolescents (étudiants du niveau secondaire, entre 12 et 18 ans, en NouvelleZélande) sur leur intention de chercher de l'aide professionnelle. D'après leur hypothèse, l'idéation suicidaire allait fonctionner comme un facteur d'approche, motivant ces individus à rechercher du secours, tout comme la détresse psychologique d'ailleurs. Mais contrairement à cette hypothèse, ils se sont rendus compte que plus l'idée du suicide était sérieuse, moins ces individus étaient portés à chercher de l'aide, l'idée agissant comme un facteur d'évitement. Ce résultat s'accorde avec une étude menée par Rudd et al. (1995), selon laquelle les patients psychiatriques suicidaires étaient en état de "négation d'aide ". On définit la négation d'aide comme "le refus d'accepter ou d'avoir accès aux ressources d'aide disponibles, en tant que fonction possible de la manifestation du désespoir du patient, de son pessimisme et de son cynisme " (Rudd et al., 1995, cité dans Carlton et Deane, 2000 : 36). Deane et al. (2000) ont constaté que les prisonniers aux prises avec des idées suicidaires étaient moins portés à chercher de l'aide que lorsqu'ils avaient d'autres problèmes personnels-émotionnels (cité dans Carlton et Deane, 2000). Il existe donc une certaine évidence à l'effet que l'état suicidaire peut exercer une influence sur la disposition à rechercher ou à recevoir de l'aide.

Finalement, à partir des ouvrages sociologiques sur la vie en prison, on peut émettre l'hypothèse qu'il est important de considérer les effets secondaires potentiellement négatifs du fait de rechercher ou d'accepter du soutien social en prison. Bien que le soutien social puisse « amortir

4. Récemment, Snow (2000) a fait une évaluation des Listener Schemes dans cinq prisons régionales. Snow a constaté que ceux qui utilisaient cette ressource pour discuter de leurs problèmes étaient « plus susceptibles de révéler leurs difficultés de relations, de harcèlement, d'acceptation de leur sentence et d'inquiétude devant leur imminente comparution devant la cour » (p. 117). Bien qu'on ne rapporte aucune différence entre utilisateurs et non utilisateurs quant à l'étendue de leur recours à des ressources de soutien externes, les utilisateurs avaient plus tendance à discuter de leurs problèmes avec des amis et avec le personnel à l'intérieur de la prison. Snow (2000) conclut que "plus d'utilisateurs avaient déclaré avoir des idées de suicide et/ou d'automutilation : 21 pour cent comparativement à 5 pour cent chez les non utilisateurs" (p. 114). On ne dispose d'aucun chiffre sur le pourcentage d'individus suicidaires qui recherchent de l'aide auprès des Listeners et le pourcentage de ceux qui sont suicidaires mais qui préferent ne pas y avoir recours. 
le stress ", aider à ajuster les émotions et mener à une amélioration de l'adaptation à la prison, le fait d'accepter du soutien peut aussi révéler la vulnérabilité du prisonnier et avoir des conséquences sur la position du prisonnier dans la hiérarchie informelle qui existe. Chercher de l'aide constitue un comportement qui signifie ouvertement que ce prisonnier souffre de détresse psychologique; reconnu alors comme une proie facile (ou plus facile), ce prisonnier peut devenir une cible de représailles pour les autres détenus. Les ouvrages sociologiques ont à ce propos l'avantage de mettre en lumière les différences de niveaux de participation dans les communautés carcérales (Clemmer, 1940 ; Sykes et Messinger, 1960 ; Wheeler, 1961 ; Bowker, 1971). On remarquera par exemple, dans ces communautés, leur tendance à attirer et à exclure des membres. En psychologie, quand un mécanisme clé sert à éliminer des membres, on parle d'ostracisme (Williams, 1997a). Voilà un domaine utile qui pourrait servir à rapprocher mécanismes sociologiques et mécanismes psychologiques. Comment et pourquoi des prisonniers sont-ils exclus des réseaux de soutien social qui émergent dans les établissements pénitentiaires?

\section{Décommunication et ostracisme en prison}

La mort sociale s'installe lorsque l'institution [...] perd son intérêt pour l'individu comme être bumain et le traite comme un simple corps - c'est-à-dire comme s'il était déjà mort (Shneidman, $1973: 159)$.

Selon Gallo et Ruggiero, la prison est un monde de "décommunication " et d'exclusion. Ceci peut être une caractéristique de la vie interne de la prison, tout comme sa fonction principale. Par ostracisme, on entend "l'exclusion d'un individu ou d'un groupe des autres individus ou groupes » (Williams, 1997a : 142). Lorsque cela survient fréquemment, son impact sur la vie, l'adaptation et le comportement suicidaire en prison est très puissant. Le fait d'être emprisonné signifie un macroniveau d'ostracisme : le prisonnier est exclu de la société dans son ensemble ${ }^{5}$. Une fois le prisonnier dirigé vers une prison et la sentence commencée, l'ostracisme est sujet à se développer à un microniveau, interpersonnel. Dès qu'ils sont emprisonnés, les individus sont sujets à être traités comme des " numéros » ou comme des " corps ", vus comme

5. Gallo et Ruggiero soutiennent que, «aux yeux de la société, ce n'est pas l'offense, mais la sanction [...] qui est chargée du véritable stigmate " (1991:289). 
des objets plutôt que comme des êtres sociaux, capables de créer leurs propres univers sociaux. Au sein même de la prison, les individus peuvent être ostracisés par les autres détenus et par le personnel, alors qu'à l'extérieur ils sont ignorés et exclus par les membres de leurs réseaux sociaux existants.

Bien qu'on ait mené des recherches sur le harcèlement dans le cadre des prisons (voir la recension de Ireland, 2000), aucune étude n'a encore examiné la fréquence ou la signification de l'ostracisme social en prison. Le modèle proposé par Williams (1997a) pour étudier l'ostracisme dans l'ensemble de la communauté peut aider à comprendre l'ostracisme en milieu carcéral. Williams (1997a) a mis de l'avant un modèle besoinmenace d'ostracisme, suggérant quatre dimensions selon lesquelles l'ostracisme peut varier : visibilité, motif, quantité et clarté causale. On soutient que les individus ostracisés se retrouvent privés de quatre besoins fondamentaux, soit l'appartenance, l'estime de soi, le contrôle et une existence utile. À court terme, on avance que les réactions au fait d'être ostracisé « comprennent sentiment de blessure, mauvaise humeur et excitation physiologique " (Williams et Zadro, sous presse : 15), avec des tentatives de regagner l'estime de soi et ainsi de suite. En ce qui concerne les effets a long terme :

les besoins menacés vont être intériorisés; un manque pralongé d'appartenance peut provoquer l'impression de n'appartenir à aucun milieu, la menace constante posée à l'estime de soi peut contribuer à la dégradation de la confiance en soi qui entraine une faible estime de soi chronique, une perte de contrôle prolongée sur l'environnement et [...] est susceptible de mener à une impuissance acquise; de plus, le sentiment d'avoir un but, irrémédiablement amoindri, peut alors forcer les gens à douter du but et de la valeur de leur existence (Williams and Zadro, sous presse : 12 )

Dans les mots d'un détenu ayant tenté de se suicider : " [C'était] de la dépression et de la rage. Je n'arrêtais pas de penser à ma famille, mes amis, à ce qui se passait autour de moi, et comme je n'avais pas reçu de lettres depuis une semaine ou deux, jai simplement pensé qu'il n'y avait pas de raison que je continue à exister puisque personne ne se souciait de moi ( (Liebling, 1995 : 177). Biggam et Power ont trouvé que les victimes de harcèlement avaient plus tendance à présenter des solutions non pertinentes et passives à leurs problèmes, pensaient que la solution exigerait plus d'effort et démontraient une plus grande détresse psychologique que les harceleurs. À une diminution de la capacité à résoudre les problèmes correspondait une augmentation de la détresse psychologique (Biggam et 
Power, 1999c). Être la cible d'ostracisme constitue la manifestation suprême du manque de soutien social. Un individu peut souffrir de manque de soutien social parce que celui-ci n'est pas disponible, alors que l'individu ostracisé est intentionnellement privé d'une relation de soutien. L'ostracisme en prison peut affaiblir la capacité d'adaptation et augmenter la vulnérabilité au stress de l'emprisonnement. En d'autres mots, cet ostracisme peut s'intégrer à « l'échelle » ou, plus dynamiquement, à la " spirale » idéation, sentiment et geste suicidaires.

On peut représenter l'impact de l'ostracisme social et du soutien social sur le comportement suicidaire sous forme de modèle. Comme nous le soutenions déjà ci-dessus, il peut être utile d'illustrer le cheminement suicidaire (Eldrid, 1998 ; "échelle du suicide " de Eldrid, in Liebling, 1999) comme une spirale plutôt que de façon linéaire. Au schéma 1 , on pourra remplacer l'échelle par une spirale. Qu'un individu s'engouffre ou non dans cette spirale dépend de l'équilibre entre les facteurs de prédisposition (incluant antécédents, expérience, manque de soutien social, faible intelligence émotionnelle, faible ajustement des émotions, faible capacité d'adaptation, faible estime de soi, perspectives limitées, délinquance, traitement psychiatrique, carences sociales et économiques) et les facteurs de protection (incluant soutien social disponible, usage constructif du temps en prison, fort ajustement des émotions, bonne capacité d'adaptation, espoirs et plans d'avenir). On pourra considérer le fait de demander ou de se faire offrir du soutien social comme un mécanisme de protection qui éloigne l'individu du " seuil suicidaire », alors que le manque de soutien social pourra être un mécanisme d'accélération du cycle, réagissant avec le système cognitifémotionnel et rapprochant l'individu du seuil du comportement suicidaire. On pourra considérer l'impact préjudiciable du manque de soutien social comme un manque "chronique " ou comme un "déclen-cheur situationnel». Bien que le manque de soutien social puisse prédisposer l'individu, cette carence conserve son impact négatif tout au long du processus suicidaire. Comme on peut l'observer au schéma 1, si un individu s'enfonce assez profondément dans ia spirale, il risque une «mort sociale », conséquence d'un ostracisme prolongé, ostracisme qui peut même être auto-infligé, ayant pour effet d'isoler l'individu. Rendu à ce point, même si un quelconque soutien social lui est offert, l'individu suicidaire pourrait refuser ou ne pas prendre conscience du soutien mis à sa disposition. En effet, un individu, ainsi que nous l'avons soutenu précédemment, 
SCHÉMA 1

Vers un modèle théorique du cheminement suicidaire des prisonniers

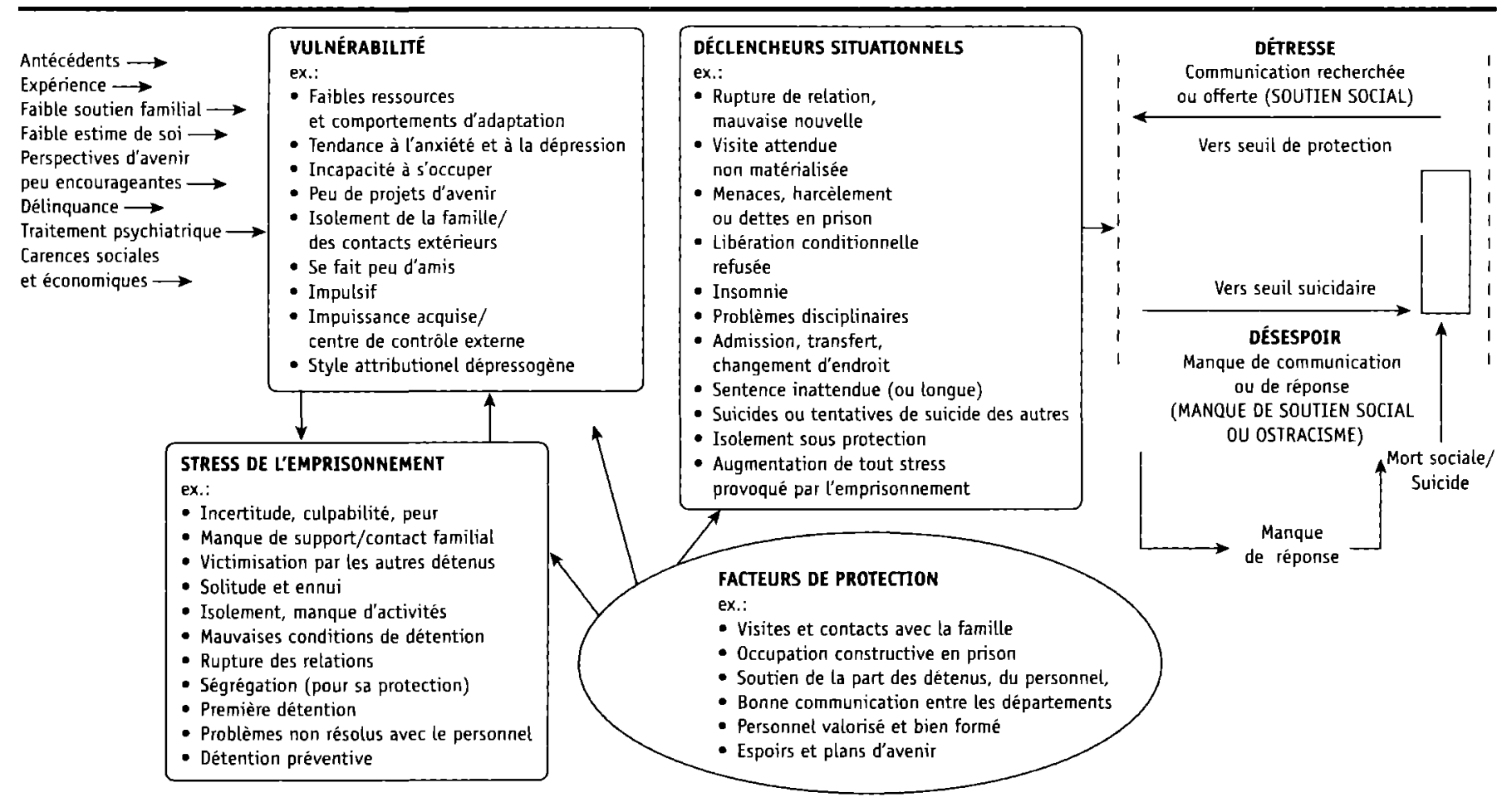


pourrait souffrir d'un manque de soutien social parce qu'il refuse de chercher ou d'accepter ce qu'on lui offre.

Ce modèle admet que la perception qu'a l'individu du niveau de soutien à sa disposition est capitale quand on en évalue l'impact sur le comportement. L'étude de Williams sur des individus qui avaient tenté de se suicider révèle qu'ils percevaient de l'abandon et du rejet là où il n'en existait probablement pas (Williams,1997b). D'autres individus peuvent réellement souffrir de rejet: Williams soutient que, si un individu se perçoit comme inférieur, raté et " méritant le rejet », il pourra en venir à créer pour lui-même cet environnement de punition ou de rejet. Ce modèle est dynamique en ce sens que les relations interpersonnelles sont en constante mutation, à la fois positivement et négativement. Selon les changements dans la microsociété de l'individu, l'aide peut lui être offerte ou retirée à tout moment. Ce modèle reconnaît également que la réponse (ou son manque) de l'institution au comportement suicidaire peut contribuer à l'engouffrement de l'individu dans la spirale, l'incitant à poser les gestes suicidaires. Il est essentiel de comprendre le processus de communication et de réponse propre au cheminement suicidaire de chaque individu afin de comprendre l'impact du soutien social et de son manque.

\section{Conclusions}

Au cours de la dernière décennie, la recherche a été assez abondante sur les suicides et les tentatives de suicide dans les prisons à travers le monde. Plusieurs études cherchent encore à établir un profil suicidaire à partir de notes prises aux dossiers d'individus décédés par suicide, ou d'entretiens réalisés avec des détenus qui avaient tenté de se suicider. Certaines études ont eu recours à des tests psychologiques et des mesures de résolution de problèmes pour arriver à préciser ces diffétents concepts. Bien qu'elles soient de nature déductive, cherchant à vérifier des hypothèses plutôt qu'à rassembler de l'information descriptive, elles restent décontextualisées. Par conséquent, elles ne parviennent pas à "fournir de l'information relativement à la signification personnelle de la pensée, de l'élaboration et de l'acte suicidaires " (Westefeld et al., $2000: 465$ ). Westefeld et al. concluent que " plutôt que d'avoir une orientation théorique, la recherche contemporaine en suicidologie continue de se pencher de façon pragmatique sur l'identification des variables reliées au suicide qui serviront de facteurs d'évaluation et de prédiction " (Westefeld et al., 2000:250). 
Dans le présent article, nous avons soutenu que l'une des approches à la compréhension du comportement suicidaire consistait à le situer dans un cadre théorique élargi, incluant les relations sociales interpersonnelles, et à puiser plus systématiquement dans la littérature sociologique disponible sur la vie sociale en prison. De cette façon, on ne considère plus que le suicide survient de nulle part (un problème de l'individu) pour le situer dans le contexte de l'univers social du détenu. Le soutien social est bidirectionnel : d'abord, en tant que facteur " protecteur ", il protège l'individu de l'impact des événements stressants ; d'autre part, lorsqu'il fait défaut, ce soutien peut être interprété comme un agent de stress en soi.

Pour examiner le comportement suicidaire dans un cadre de relations sociales, nous aurons besoin d'un programme plus varié et intégré, incluant recherche ethnographique, entretiens et groupes de discussion, en plus d'autres travaux de même nature que ceux exposés ici ${ }^{6}$. Les ethnographies classiques dans le domaine de la recherche sur le suicide ont exploré les herméneutiques ou les constructions explicites du suicide, par le biais de rapports officiels et d'histoires de cas (Bailey, 1998 ; voir aussi Douglas, 1967 ; Atkinson 1982 ; Taylor, 1982). Nous favorisons une approche ethnographique à l'étude de l'idéation du comportement suicidaire ainsi que des processus d'adaptation et de soutien social en prison. Nous privilégions une telle approche qualitative afin d'obtenir une appréciation significative des univers et des combats ou « contextes microsociaux » (Bailey, 1998 : 24) des gens qui vivent en détention. Peut-on parler de " camouflage de vulnérabilité "? Est-ce que les prisonniers suicidaires tentent de se créer une identité publique "de dur qui s'adapte ", incompatible avec leur identité privée d'individu vulnérable en proie à de la souffrance psychologique? Quelle est l'importance de l'apport de soutien et de ses sources? Quels rôles s'attribuent les familles, le personnel de l'institution et les Listeners? Au cœur de toute recherche, on devrait retrouver une détermination de comprendre la signification subjective du suicide pour tous ceux qui vivent au sein de la communauté carcérale. Si le suicide est l'affaire de tous, alors on devrait considérer l'expérience de toutes les personnes

6. L'ethnographie est " une description et une interprétation d'un groupe culturel ou social, ou d'un système [et] implique l'observation prolongée du groupe, tout particulièrement par l'observation participante par laquelle le chercheur s'immerge dans la vie quotidienne du peuple ou par le moyen d'entretiens en tête à tête avec les membres du groupe " (Creswell, 1998: 58). 
concernées et la toile complexe des relations qui constituent la communauté en prison devrait être l'objet d'un examen systématique.

\section{Références}

AtKinson, J.M.1982. Discovering Suicide. Studies in the Social Organisation of Sudden Death, Londres : McMillan.

BACKETT, S. 1987. " Suicides in Scottish Prisons " British Journal of Psychiatry $151: 218-221$.

BaCketT, S. 1988. "Suicide and Stress in Prison" in Imprisonment Today, sous la direction de S. Backett, J. McNeil et A. Yellowlees. Londres : McMillan.

Bailey, V. 1998. This Rash Act. Suicide Across the Life Cycle in the Victorian City, Stanford : Stanford University Press.

BEN-DAvid, S. et SILFEN, P. 1994. "In Quest of a Lost Father? Inmate's Preferences of Staff Relations in a Psychiatric Prison Ward "International Journal of Offender Therapy and Comparative Criminology 38 : 131-139.

BenezeCH, M. 1999. "Suicide and its Prevention in French Prisons " Annales Médico-Psychologiques 157 (8) : 561-566.

Biggam, F.H. et Power, K.G. 1997. "Social Support and Psychological Distress in a Group of Incarcerated Young Offenders " International Journal of Offender Therapy and Comparative Criminology $413: 213-230$.

Biggam, F.H. et Power, K.G. 1999a. a A Comparison of the Problem-Solving Abilities and Psychological Distress of Suicidal, Bullied, and Protected Prisoners » Criminal Justice and Behavior 26 : 196-216.

BlgGam, F.H. et Power, K.G. 1999b. "Suicidality and the State-Trait Debate on Problem Solving Deficits: A Re-examination with Incarcerated Young Offenders" Archives of Suicidal Research $5: 27-42$.

Biggam, F.H. et Power, K.G. 1999c. " Social Problem-Solving Skills and Psychological Distress among Incarcerated Young Offenders: The Issue of Bullying and Victimisation " Cognitive Therapy and Research 23:307326.

Bogue, J. et Power, K. 1995. "Suicide in Scottish Prisons 1976-93 " The Journal of Forensic Psychiatry 63 : 527-540.

BONNER, R.L. 1992. "Isolation, Seclusion, and Psychosocial Vulnerability as Risk Factors for Suicide Behind Bars ", Pp. 398-419 in Assessment and Prediction of Suicide, sous la direction de R. Maris, A.L. Berman, J.T. Maltsberger et R.I. Yufit. New York, NY : The Guilford Press.

BONNER, R.L. et RICH, A.R. 1990. "Psychosocial Vulnerability, Life Stress, and Suicidal Ideation in a Jail Population : A Cross-validation Study » Suicide and Life-Threatening Bebavior 20 (3) : 213-224.

BONNER, R.L. 2000. « Correctional Suicide Prevention in the Year 2000 and Beyond » Suicide and Life-Threatening Behavior 30 (4) : 370-376.

BourgolN, N. 1993. "Suicides in Prisons " Population 483 : 609-625. 
BuRTCH, B.E. et ERICSON, R.V. 1979. The Silent System : An Inquiry into Prisoners who Suicide and Annotated Bibliography, Centre of Criminology : University of Toronto.

Camus, A. 1955. The Myth of Sisyphus and Other Essays, Londres : Hamish Hamilton.

Carlton, P.A. et Deane, F.P. 2000. "Impact of Attitudes and Suicidal Ideation on Adolescents' Intentions to Seek Professional Psychological Help " Journal of Adolescence 23 : 35-45.

Clemmer, D. 1940. The Prison Community, New York : Holt, Rinehart 8 Winston.

COHEN, S. et TAYLOR, L. 1972. Psychological Survival, Harmondsworth : Penguin.

CRESWELL, J.W. 1998. Qualitative Inquiry and Research Design. Choosing Among Five Traditions, Londres: Sage.

Crighton, D. et Towl, G. 1997. "Self-inflicted Deaths in Prisons in England and Wales: An Analysis of the Data for 1998-90 and 1994-95 " Issues in Criminological and Legal Psychology $28: 12-20$.

DaltoN, V. 1999. "Suicide in Prison 1980 to 1988 : An Overview " Trends and Issues in Crime and Criminal Justice. Disponible à http ://www.aic.gov.au.

DANTO, B. 1972. Jail House Blues : Studies of Suicidal Bebavior in Jail and Prison, Orchard Lane, Mich. : Epic.

DEAR, G.E. 2000. «Functional and Dysfunctional Impulsivity, Depression, and Suicidal Ideation in a Prison Population " Journal of Psychology 134 (1) $: 77-80$.

DeAR, G.E., Thomson, D.M. et Hills, A.M. 2000. "Self-harm in Prison. Manipulators can also be Suicide Attempters "Criminal Justice and Behavior $27: 160-175$.

Dear, G.E., Thomson, D.M., Hall, G.J. et Howells, K. 1998. « Self-inflicted Injury and Coping Behaviours in Prison ", Pp. 189-199 in Suicide Prevention: The Global Context, sous la direction de R. J. Kosky, H. S. Eshkevari, R. Hassan et R. Goldney. New York : Plenum Press.

Dear, G.E., Thomson, D.M., Howells, K. et Hall, G.J. (sous presse). "Self-harm in Western Australian Prisons : Differences between Prisoners who have Self-harmed and those who have not ».

DOOLEY, E. 1990. “ Prison Suicide in England and Wales, 1972-1987 " British Journal of Psychiatry $156: 40-45$.

DOUGLAS, J. 1967. The Social Meanings of Suicide, Londres : Routledge.

Du Rand, C.J., Burtka G.J., Federman, E.J., Haycox, J. A. et Smith, J.W. 1995. "A Quarter of Suicide in a Major Urban Jail : Implications for Community Psychiatry » American Journal of Psychiatry 152: 1077-1080.

Dur K Heim, E. 1897/1952. Suicide. A Study in Sociology, Londres : Routledge.

ELDRID, J. 1988. Caring for the Suicidal. Londres : Constable.

ESKIN, M. 1995. « Suicidal Behavior as Related to Social Support and Assertiveness among Swedish and Turkish High School Students " Journal of Clinical Psychology 11: 153-158. 
Fulwiler, C., Forbes, C., Santangelo, S.L. et Folstein, M. 1997. « Selfmutilation and Suicide Attempt: Distinguishing Features of Prisoners » Journal of the American Academy of Psychiatry and Law $25: 69-77$.

Gallo, E. et Ruggiero, V. 1991. "The 'Immaterial' Prison : Custody as a Factory for the Manufacture of Handicaps » International Journal of the Sociology of Law 19 : 273-291.

Green, C., Kendal, K., Andre, G., Looman, T. et Polvi, N. 1993. « A Study of 13 Suicides among Canadian Federal Prisoners" Medicine, Science, and the Law 332:121-127.

Haines, J. et Williams, C.L. 1997. " Coping and Problem-solving of Selfmutilators " Journal of Clinical Psychology 53 : 177-186.

HAYES, L.M. 1995. Prison Suicide: An Overview and Guide to Prevention, Washington, DC : U.S. Justice Department, National Institute of Corrections.

Home OfFICE. 1999. Suicide is Everyone's Concern. A Thematic Review by HM Chief Inspector of Prisons for England and Wales, Londres : Home Office.

Houses OF PARLIAMENT. 1999. Saving Lives: Our Healthier Nation, White Paper, Londres : The Stationery Office.

InCH, H., RowlandS, P. et Soliman, A. 1995. "Deliberate Self-harm in a Young Offenders' Institution » The Journal of Forensic Psychiatry 61:161171.

IRELAND, J.L. 2000. " "Bullying" among Prisoners : A Review of Research " Aggression and Violent Behavior 5 : 201-215.

IRWIN, J. et CRESSEY, D.R. 1962. “Thieves, Convicts and the Inmate Culture » Social Problems 10 (1) : 142-155.

IVANOFF, A. et JANG, S.J. 1991. "The Role of Hopelessness and Social Desirability in Predicting Suicidal Behavior : A Study of Prison Inmates " Journal of Consulting and Clinical Psychology 59 : 394-399.

IVANOFF, A. 1992. "Background Risk Factors Associated with Parasuicide among Male Prison Inmates " Criminal Justice and Behavior 19 : 426-436.

IVanoff, A., Smyth, N.J., Grochowski, S., Jang, S.J. er Klein, K.E. 1992. "Problem Solving and Suicidality among Prison Inmates: Another look at State versus Trait » Journal of Consulting and Clinical Psychology $60: 970$ 973.

JACK, R. 1992. Women and Attempted Suicide, Hillsdale : Lawrence Erlbaum Associates.

Joukamma, M. 1997. "Prison Suicide in Finland, 1969-1992 "Forensic Science International 89 (3) : 167-174.

Lazarus, R.S. et Folkman. 1984. Stress, Appraisal and Coping, New York: Springer.

Lazarus, R.S. 1966. Psychological Stress and the Coping Process, New York: McGraw-Hill.

LAZARUS, R.S. 2000. «Evolution of a Model of Stress, Coping and Discrete Emotions ", Pp. 195-222 in Handbook of Stress, Coping, and Health. Impli- 
cations for Nursing Research, Theory and Practice, sous la direction V. Hill Rice. London : Sage.

LESTER, D. et DANTO, B. 1993. Suicide Behind Bars: Prediction and prevention, Philadelphia : Charles Press.

Liebling, A. et Krarup, H. 1993. Suicide Attempts in Male Prisons, Londres : Home Office.

LIEBLING, A. et PRICE, D. 1999. An Exploration of Staff-Prisoner Relationships at HMP Whitemoor, Prison Service Research, Report No. 6.

Liebling, A. et WaRd, T. 1995. "Prison Doctors and Prison Suicide Research " in The Health of Prisoners, sous la direction de R. Cresse, W.F. Bynum et J. Bearn. Atlanta.

Liebling, A. 1992. Suicides in Prison, Londres : Routledge.

LIEBLING, A. 1995. "Vulnerability and Prison Suicide " The British Journal of Criminology 35 (2) : 173-187.

Liebling, A. 1999. "Prison Suicide and Prisoner Coping », Pp. 283-359 in Crime and Justice : A Review of Research vol. 26, sous la direction de M. Tonry and $J$. Petersilia.

Liebling, A. 2000. "Prison Officers, Policing and the Use of Discretion " Theoretical Criminology 4 (3) : 333-359.

MATHIESEN, T. 1965. The Defences of the Weak: A Sociological Study of a Norwegian Correctional Institution, Londres : Tavistock.

NEGY, C., WOODS, D.J. et CARLSON, R. 1997. "The Relationship between Female Inmates' Coping and Adjustment in a Minimum-Security Prison " Criminal Justice and Behavior 24 (2) : 224-233.

SAFER CuStOdy Group. 2001. Disponible à http ://www.hmprisonservice.gov.uk/

SChaller, G., ZimmermanN, C. et Raymond, L. 1996. "Suicidal Gestures (including wrist slashing) in a Swiss Prison: Identifying the Risk Factors »Sozial-Und Praventivmedizin 41 (4) : 249-256.

SChotte, D.E. et Clum, G.A. 1982. "Suicidal Ideation in a College Population : A Test of a Model "Journal of Consulting and Clinical Psycho$\log y 50$ (5) : 690-696.

SChotTe, D.E. et Clum, G.A. 1987. « Problem-Solving Skills in Suicidal Psychiatric Patients " Journal of Consulting and Clinical Psychology 55 (1) : 49-54.

Shapira, R. et NAvon, D. 1985. "Staff-Inmate Co-operation in Israeli Prisons : Towards a Non-Functionalist Theory of Total Institutions " International Review of Modern Sociology 15:131-146.

Smyth, N.J., Ivanoff, A. et Jang, S.J. 1994. "Changes in Psychological Maladaptation among Inmate Parasuicides " Criminal Justice and Behavior $21: 357-365$.

SNOw, L. 2000. "The Role of Formalised Peer-Group Support in Prisons ", Pp. 102-120 in Sucide in Prisons, sous la direction de G. Towl, L. Snow et M. McHugh. Leicester : BPS Books. 
SYkes, G et MESSINGER, S. 1960. "The Inmate Social System ", Pp. 5-19 in Theoretical Studies in Social Organisation of the Prison. New York : Social Science Research Council.

SYKeS, G. 1958. The Society of Captives, Princeton, N.J. : Princeton University Press.

Tatarelli, R., Mancinelli, I, TagGi, F. et Polidori, G. 1999. "Suicide in Italian Prisons in 1996 and 1997. A Descriptive Epidemiological Study " International Journal of Offender Therapy and Comparative Criminology 43 (4) : 438-447.

TAYLOR, S. 1982. Durkbeim and the Study of Suicide, Londres: Macmillan.

TOPP, D.O. 1979. « Suicide in Prison » British Journal of Criminology 143 : 24-27.

TowL, G. 1999. "Self-inflicted Deaths in Prisons in England Wales from 1988 to 1996 " in Suicides in Prisons. Research, Policy and Practice, sous la direction de G. Towl, M. McHugh et D. Jones. Brighton: Pavilion $\mathrm{Pu}-$ blishing.

Underwood, P.W. 2000. "Social Support : The Promise and the Reality ", Pp. 367-391 in Handbook of Stress, Coping, and Health. Implications for Nursing Research, Theory and Practice, sous la direction de V. Hill Rice. Londres : Sage.

VAN AKen, M.A.G., van Lieshout, C.F.M., SCholte, R.H.L. et BRanje, S.J.T. 1999. "Relational Support and Person Characteristics in Adolescence " Journal of Adolescence $22: 819-833$.

Westefeld, J.S., RANGE, L.M., Rogers, J.R., MAPLES, M.R., BROMLEY, J.L. et ALCORN, J. 2000. "Suicide : An Overview " The Counseling Psychologist $28(4): 445-510$.

WheELER, S. 1961. "Socialization in Correctional Communities " American Sociological Review $26: 697-712$.

White, J., LegGetT, J. et BeECH, A. 1999. «The Incidence of Self-Harming in the Male Population of a Medium-Secure Psychiatric Hospital » The Journal of Forensic Psycbiatry 10 (1) : 59-68.

Wichmann, C., Serin, R. et Motiuk, L. 2000. Predicting Suicide Attempts among Male Offenders in Federal Penitentiaries, Research Branch, Correctional Service Canada.

Williams, K.D. et Zadro, L. (sous presse). « Ostracism : On Being Ignored, Excluded and Rejected » in Rejection, sous la direction de M. Leary. New York : Oxford Press.

Williams, K.D. 1997a. "Social Ostracism », Pp. 133-170 in Aversive Interpersonal Bebaviour, sous la direction de R. Kowalski. New York : Plenum.

Williams, M. 1997b. Cry of Pain: Understanding Suicide and Self-Harm. Londres : Penguin.

World Health Organisation. 1999. Health in Prisons Project, disponible à http :/ www.hipp-europe.org 